\title{
Efficacy of Isotonic Seawater Solution on Inflammation of the Ocular Surface in Matrix Metalloproteinase MMP-9-Positive Dry Eye Disease Patients
}

\author{
Diaz-Llopis Manuel1,2,3,4,5, Pinazo-Duran Maria Dolores,3,3,7, Diaz-Guiñon Loreto5, \\ Rahhal-Ortuño Miriam ${ }^{8}$, Gallego-Pinazo Roberto9, Dolz-Marco Rosa9, \\ Diaz-Guiñon Teresa5, Diaz Maria ${ }^{5}$, Romero Francisco Javier ${ }^{4}$ \\ ${ }^{1}$ University of Valencia, Valencia, Spain \\ ${ }^{2}$ Ophthalmology Unit, Faculty of Medicine, University of Valencia, Valencia, Spain \\ ${ }^{3}$ Molecular Ophthalmology Unit, Faculty of Medicine, University of Valencia, Valencia, Spain \\ ${ }^{4}$ Consellería de Sanitat (Valencian Regional Health Ministry), Valencian Autonomous Government, Valencia \\ ${ }^{5}$ IOVA-Valencia Ophthalmology Institute, Valencia, Spain \\ ${ }^{6}$ Doctor Peset University Hospital, Valencia, Spain \\ ${ }^{7}$ Santiago Grisolía Eye Research Laboratory, FISABIO, Valencia, Spain \\ ${ }^{8}$ Department of Ophthalmology, La Fe Hospital, Valencia, Spain \\ ${ }^{9}$ IMED Hospital, Valencia, Spain \\ Email:manuel.diaz@uv.es,pinazoduran@yahoo.es, loretodiazg@hotmail.com,miriamrahhal@hotmail.com, \\ robertogallegopinazo@yahoo.es, rousss@hotmail.com, teresadiazg@hotmail.com,dizadiaz@gmail.com, \\ jromerogomez@gmail.com
}

How to cite this paper: Manuel, D.-L. Dolores, P.-D.M., Loreto, D.-G., Miriam, R.-O., Roberto, G.-P., Rosa, D.-M., Teresa, D.-G., Maria, D. and Javier, R.F. (2020) Efficacy of Isotonic Seawater Solution on Inflammation of the Ocular Surface in Matrix Metalloproteinase MMP-9-Positive Dry Eye Disease Patients. Open Journal of Ophthalmology, 10, 211-219.

https://doi.org/10.4236/ojoph.2020.103023

Received: June 15, 2020

Accepted: August 3, 2020

Published: August 6, 2020

\begin{abstract}
Purpose: Prospective study to evaluate the anti-inflammatory efficacy of isotonic seawater solution on metalloproteinase 9 (MMP-9) levels in the tears of patients with dry eye disease (DED). Methods: A total of 50 patients were included, with the following dry eye criteria in both eyes: InflammaDry ${ }^{\circledR}$ test initially positive, ocular surface disease index (OSDI) score $\geq 12$ and $<33$, tear film breakup time (TBUT) $\leq 10$ seconds, Shirmer I test result $\leq 10 \mathrm{~mm} / 5$ minutes, corneal staining $\geq 1$. Additionally, all the patients selected corresponded to a dry eye type 2 - 3 severity degree - mild and moderate - Dry Eye Workshop Study (DEWS) classification. The InflammaDry test is used to measure levels of MMP9 at the beginning and the end of the study. All patients were treated exclusively with isotonic seawater solution 5 times a day for 3 weeks. Results: The InflammaDry test was positive in $100 \%$ of the patients $(\mathrm{n}=50)$ and in $100 \%(\mathrm{n}=100)$ of the eyes before treatment. In 14 (28\%) patients the test became negative in both eyes, and in $15(30 \%)$ it was
\end{abstract}


Copyright (C) 2020 by author(s) and Scientific Research Publishing Inc. This work is licensed under the Creative Commons Attribution International License (CC BY 4.0).

http://creativecommons.org/licenses/by/4.0/ negative in one of the eyes. In $43 \%(n=43)$ of the positive eyes, MMP-9 became undetectable in the tear fluid following treatment with isotonic seawater. This change was statistically significant $(\mathrm{p}<0.001)$. Conclusions: The washes with ophthalmic isotonic seawater solution have a significant impact on inflammation of the ocular surface in dry eye disease, with an ability to make MMP-9 levels negative in $43 \%$ of cases.

\section{Keywords}

Dry Eye, Inflammadry Test, MMP-9, Ocular Surface Inflammation, Ophthalmic Isotonic Seawater Solution

\section{Introduction}

Dry eye disease (DED) is a multifactorial pathology in which inflammation plays an important role in the physiopathology of the disease [1]. Of the various proinflammatory cytokines implicated, such as interleukin IL-1beta, IL-6, tumour necrosis factor-alpha (TNF-alpha), interferon IFN gamma, caspase, transglutaminase 2 and matrix metalloproteinase MMP-3, etc., MMP-9 plays a fundamental role in the start, progression and creation of the vicious inflammatory circle that leads to chronic perpetuation of the disease, as well as to secondary lesions and irreversible damage to the corneal and conjunctival epithelial cells (clinical keratoconjunctivitis) [2] [3] [4].

InflammaDry is a non-invasive commercial test that allows for the simple, accurate and rapid measurement of tear MMP-9 levels on an outpatient basis. It positively identifies levels greater than $40 \mathrm{ng} / \mathrm{ml}$ [5]. Several studies have shown a correlation between tear MMP-9 levels and the various clinical and anatomical parameters of dry eye, especially the Ocular surface disease index (OSDI), conjunctival staining and corneal staining. Several studies have also demonstrated a significant reduction in tear MMP-9 levels, indicated by a negative InflammaDry test, following different anti-inflammatory treatments such as corticosteroids, tacrolimus and topical cyclosporine [6] [7].

In a previous study, our team demonstrated the efficacy of ophthalmic isotonic seawater solution in improving the symptoms, corneal and conjunctival lesions, as well as in significantly reducing levels of the proinflammatory molecules IL1 and IL6 in DED patient tears, showing it to be globally more efficient that treatment with carmellose artificial tears [8].

The proposed study aims to establish the anti-inflammatory efficacy of eyewashes with isotonic seawater, showing them to be capable of making tear MMP-9 levels negative or undetectable in patients with DED.

\section{Methods}

\subsection{Study Design}

Prospective study was conducted between January 2017 and June 2019. The 
study was conducted in accordance with the ethical practices contained in the Declaration of Helsinki. The informed consent and clinical protocol were reviewed and approved by the University of Valencia ethical committee prior to the start of the study. A preliminary study was carried out to calculate the number of patients needed to be included in the study (XISTAT life science software and STATISTICA version 8 software). Each patient signed a specific informed consent form before the start of the study that explained the purpose of the study.

\subsection{Subjects}

The study included for each of the patients 1) general data (sex, age) and general pathologies 2) study of the anterior pole by means of slit-lamp examination 3) tonometry, and 4) retinography.

The inclusion criteria for patients with dry eye required all patients to satisfy all the following criteria in both eyes at both the screening and baseline examinations: InflammaDry test initially positive, ocular surface disease index (OSDI) score $\geq 12$ and $<33$, tear film breakup time (TBUT) $\leq 10$ seconds, Shirmer I test result $\leq 10 \mathrm{~mm} / 5$ minutes, corneal staining $\geq 1$. Only patients with DED with a severity grading of 2 and 3 (mild and moderate) were selected, according to the dry eye severity grading scheme from the Dry Eye Workshop Study (DEWS) classification, excluding severe cases (OSDI 33-100), so that the group was as homogeneous as possible [9] [10]. Patients were always selected with a similar severity grading in both eyes.

Exclusion criteria included the following: 1) the presence of any previous eye disease such as neurotrophic keratitis, keratoconus, allergic conjunctivitis, pemphigoid, etc., any previous corneal refractive surgery or any previous ocular surface surgery (pterygium, etc.); 2) use of contact lenses in the two months prior to the start of the study; 3 ) use of any type of eye drops from two months prior to the start of the study, especially lubricants, corticosteroids, tacrolimus, cyclosporine, azithromycin or tetracyclines that may alter MMP-9 levels in tears and directly interfere with the positivity or negativity of the InflammaDry test; 4) the presence of tear plugs or their removal within two months prior to the start of the study; 5) any intraocular surgery during the six months prior to the start of the study; 6) any oral pharmaceuticals especially corticoids, immunosuppressants, antihistamines, hormones, benzodiazepines, beta-blockers, tetracyclines; 7) any systemic disease, especially allergic disease in any area (rhinitis, dermatitis, asthma), Sjögren's syndrome, rheumatic and autoimmune diseases, sarcoidosis, or graft-versus-host disease.

\subsection{MMP-9 Assessment, InflammaDry test}

The InflammaDry test was used to measure levels of MMP-9. InflammaDry is capable of detecting levels of MMP-9 greater than $40 \mathrm{ng} / \mathrm{ml}$, giving only a positive or negative reading [11]. The InflammaDry test has a sensitivity of $85 \%$ and 
a specificity of $94 \%$ in the detection of pathological levels of MMP-9 in tears. Specifically, those patients in whom the InflammaDry test was negative for one of the two eyes before the start of the study were excluded.

All the tear samples were taken and the InflammaDry test (RPS, Sarasota, Florida, USA) and the collection of data was always carried out by a single experienced researcher (MDLL) so as to avoid possible variables in the evaluation of MMP-9 levels. The test was always conducted after a period of 3 hours from the last application of the treatment, and under standardised room illumination and temperature conditions (between $20^{\circ} \mathrm{C}$ and $25^{\circ} \mathrm{C}$ ).

No anaesthetic could be applied to the eye for at least 10 minutes before the collection of a sample. The lower eyelid of the patients was gently lowered to expose the lower palpebral conjunctiva, relaxing the eyelid every 3 touches of the tip to allow the patient to blink. The sample collection tip was gently applied 6 8 times to different points on the lower eyelid conjunctiva and was later held for an additional 5 seconds on the inferior conjunctival fornix. A swiping motion must never be used for collection of the samples.

The sample was placed in the cassette and the tip soaked in buffer for $20 \mathrm{sec}$ onds. After resting in a horizontal position for 10 minutes, the colour of the window was evaluated. If a blue line appeared in the window, the MMP-9 levels in the teardrop were considered negative, and if a red line appeared, the levels were considered positive.

In those cases with an invalid result-with no blue or red line-in either of the two eyes, the patient continued with the treatment and a new InflammaDry test was repeated under the same conditions 2 days later only in the eye with the previously invalid result. If the result was invalid again, the patient was excluded from the study.

\subsection{Treatment Protocol}

All the patients were treated exclusively with a cold micro-filtered isotonic seawater spray (Quinton ${ }^{\circledR}$ Eye Health supplied by Laboratories Quinton, Alicante, Spain) 5 times a day for 3 weeks (Figure 1).

The Quinton Eye Health isotonic seawater solution is a polyelectrolytic solution $\left(\mathrm{Na}^{+}, \mathrm{Cl}^{-}, \mathrm{Mg}^{2+}, \mathrm{K}^{+}, \mathrm{Ca}^{2+}, \mathrm{Cu}^{2+}, \mathrm{Zn}^{2+}, \mathrm{Be}^{2+}, \ldots\right)$ with the presence of organic nutrients such as vitamins (D-Biotin, Riboflavin, Nicotinamide, cyanocobalamin) and other biomolecules [1]. The seawater was extracted in areas of planktonic proliferation and cold micro-filtered at $0.22 \mu \mathrm{m}$ in a clean room, finally being brought to isotony by adding spring water in order to obtain a salinity analogous to that of natural tear fluid.

The isotonic solution was applied as a spray a few centimetres from the eye of the patient.

\subsection{Statistical Analysis}

For statistical comparison of the MMP-9 levels before and after treatment, 


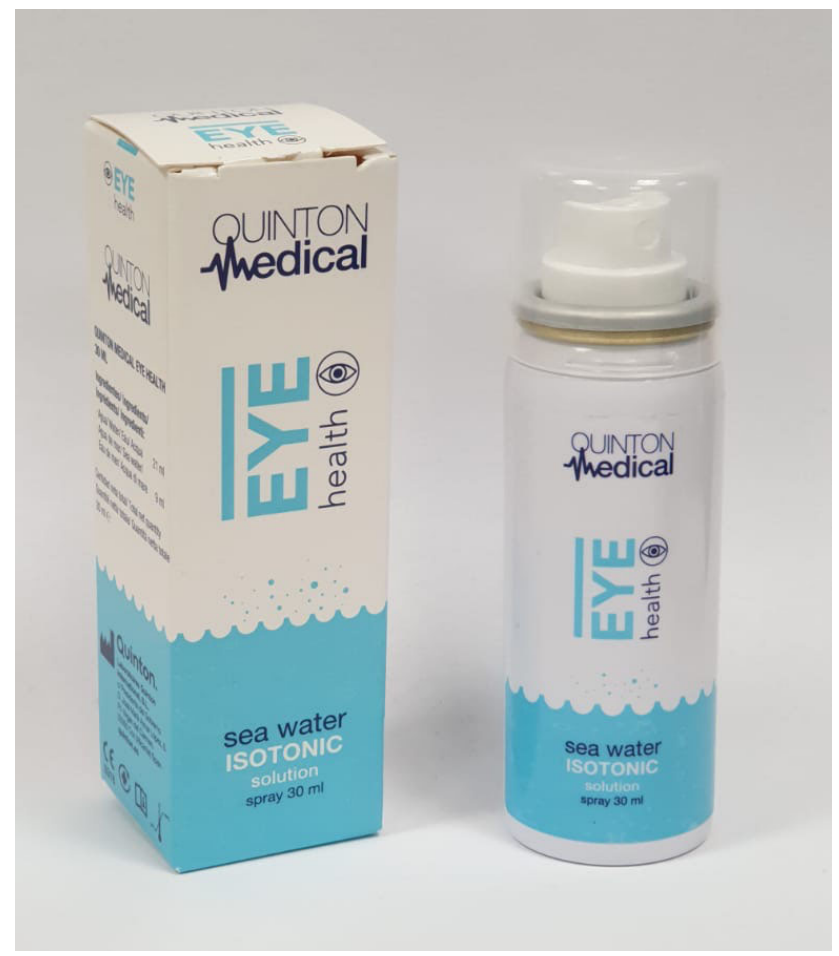

Figure 1. Quinton ${ }^{\circledR}$ ocular spray bottle.

paired t-tests and the Wilcoxon signed-rank test were conducted. The level of significance was set at $\mathrm{P}<0.01$. The data were analysed using SPSS 18.0 (SPSS, Chicago, IL, USA).

\section{Results}

A total of 1016 patients were studied in those patients who met all the inclusion criteria for this study.

\subsection{Patient Demographics}

A total of 50 participants were recruited into the study (Table 1).

All the DED patients corresponded to a type 2 - 3 degree of severity (mild \& moderate) according to the DEWS classification (9), the characteristics of which are described in Table 2.

\subsection{MMP-9 Positivity and Treatment Response}

In 6 patients with a positive InflammaDry test in both eyes previous to the start of the study, the test was invalid (absence of blue or red line) in at least one of the eyes at the end of the study. They were accordingly eliminated from the study and replaced by another 6 subjects so as to complete the series of 50 patients.

$100 \%$ of the patients $(n=50)$ and $100 \%$ of the eyes $(n=100)$ were InflammaDry-positive before starting the treatment. Following treatment with seawater, a total of 29 patients (58\%) had negative test results in at least one eye, and of 
Table 1. Demographic data of the population studied $(n=50)$.

\begin{tabular}{cc}
\hline \multicolumn{2}{c}{ Demographic data } \\
\hline Age, mean \pm SD $65.08 \pm 9.42$ years (range: $50-79$ years) \\
Female : male & $1.65: 1$ \\
Dry Eye Severity, $\mathrm{n}$ & $\mathrm{n} 33$ \\
-Mild & $\mathrm{n} 17$ \\
-Moderate & $\mathrm{n} 11$ \\
Hypertension & $\mathrm{n} 13$ \\
Cardiovascular disease & $\mathrm{n} 9$ \\
Diabetes & $\mathrm{n} 14$ \\
Osteoarthritis & $\mathrm{n} 8$ \\
Smoking history & $\mathrm{n} 21$
\end{tabular}

Table 2. Dry eye severity level.

\begin{tabular}{ccc}
\hline Dry Eye Severity Level & 2 Mild & 3 Moderate \\
\hline MMP-9 positive, \% & $100 \%$ & $100 \%$ \\
Number of patients, $\mathrm{n}$ & 33 & 17 \\
OSDI test, score & $18.17 \pm 11.83$ & $43.32 \pm 19.97$ \\
TBUT, seconds & $5.36 \pm 3.18$ & $3.42 \pm 2.16$ \\
Shirmer test, mm/5 min & $10.97 \pm 6.46$ & $6.60 \pm 3.59$ \\
Corneal staining, score 1,2 & $1.46 \pm 0.74$ & $2.48 \pm 0.68$ \\
\hline
\end{tabular}

these $14(28 \%)$ had negative test results in two eyes and $15(30 \%)$ in one of the two eyes ( 6 patients in the right eye and 9 in the left eye) representing a total of $43 \%$ of the eyes. These differences were statistically very significant $(\mathrm{p}<0.01)$.

\subsection{Side Effects}

No side effects were observed in any patient during the treatment.

\section{Discussion}

MMP-9 is an inflammatory marker that has been frequently shown to be elevated in the tears of patients with dry eyes, though not always. According to previous studies, in $30 \%-65 \%$ of the total number of diagnosed cases of DED in which MMP-9 in the tear has been measured using the InflammaDry test, it has given a positive result, as well as in $5 \%-6 \%$ of healthy control patients [12] [13]. This variability could be influenced by the different selection criteria used in each study. The number of cases of DED preselected in this study that had a positive InflammaDry test result was $53.5 \%$, and the study was conducted exclusively in the positives in order to evaluate the capacity and efficacy of seawater in 
an ophthalmic solution at making MMP-9 levels negative.

Normal MMP-9 levels $(\mathrm{ng} / \mathrm{ml})$ in human tears vary between $3 \mathrm{ng} / \mathrm{ml}$ and 40 $\mathrm{ng} / \mathrm{ml}$. The InflammaDry test has a sensitivity of $85 \%$ and a specificity of $94 \%$ in the detection of pathological levels of MMP-9 in tears [11] In previous studies, elevated levels of MMP-9 in patients with moderate to severe dry eye disease has been correlated with the severity and intensity of the symptoms and the corneal alterations of these patients, and the reduction in these with good response to the treatments [5] [6] [7]. MMP-9 plays a role in the regulation of corneal epithelial desquamation. The increase in MMP-9 activity in dry eyes contributes to disruption of the functioning of the corneal epithelial barrier, to the increase in corneal epithelial desquamation and to irregularity of the corneal surface (keratitis) and secondary ocular irritation (red eyes, gritty sensation, etc.) [2] [3] [4].

In this study, in the $43 \%$ of the eyes with dry eye and a positive InflammaDry test previous to the treatment, the test was negative following treatment with seawater alone. This efficacy is similar to that encountered with other treatments such as lifitegrast or cyclosporine + oral omega- 3 fatty acids + frequent artificial tear replacement (30\% - 54\%) [5] [12] [13].

In contrast to standard isotonic saline solution, seawater has a mineral composition which is less rich in sodium ions and much richer in bicarbonate, potassium, magnesium and calcium, as well as having a more alkaline $\mathrm{pH}$ (7.9). This can partially explain its anti-inflammatory and clinical efficacy in dry eye. Low sodium levels that reduce or eliminate the electrolyte imbalance of the ocular surface are a fundamental factor in the origin of the physiopathology chain of dry eye. Elevated bicarbonates and an alkaline $\mathrm{pH}$ that reduce the viscosity of pathological conjunctival secretions while decreasing acidification of the inflammation-damaged ocular surface promote the tropism, healing and restoration of the integrity of the conjunctival mucosa and corneal epithelium through EGF levels. High levels of calcium have a trophic effect on the epithelia. Elevated potassium and magnesium have a considerable anti-inflammatory capacity in reducing levels of TNF, IL-1 beta, IL-6, IL-8, RANTES, as well as impeding the vicious circle of dry eye chronification and perpetuation. Additionally, potassium and magnesium reduce inflammation by reducing the secretion, activation and degranulation of neutrophils, eosinophils, mastocytes and macrophages, as well as the apoptosis of conjunctival mucosa cells during inflammatory processes and healing by means of the activation of the EGF/EGFR pathway [14] [15] [16] [17].

To these mechanisms of action as described above, we must add a specific one: its of, shower-type effect, mechanically sweeping much of the cellular waste from the eye surface, and the hyperosmolarisation of electrolytes and pro-inflammatory molecules and cells (IL-1 beta, IL-6, IL-8 and MMP-3, especially MMP-9, TNF and macrophages and T lymphocytes). The efficacy of seawater has already been previously documented, in addition to DED (8), in other dry and inflammatory diseases of the human body (dermatitis, trophic ulcers of 
the skin, atopic rhinitis [14] [15] [16]. A previous study [8] showed that seawater reduces the presence of the pro-inflammatory molecules IL-1 beta and IL-6 to levels almost similar to those of the normal population without dry eye. This study shows that seawater is able to normalise previously elevated MMP-9 levels in $43 \%$ of the eyes with DED, thus providing a biochemical basis for its anti-inflammatory efficacy.

\section{Limitations}

This study has one limitation: the duration was too short and may be insufficient to assess the impact of seawater on dry eye disease in the long term, since 6-month studies are required. Even so, the results provided are valuable and justify initiating a long-term study to evaluate the effectiveness of seawater at its best and to improve the chronic course of dry eye disease.

\section{Conclusion}

This study has shown that an isotonic solution of seawater is effective in reducing the levels of the MMP-9 proinflammatory molecule in tears to an extent that is statistically significant, being able to transform the positive levels prior to treatment into $43 \%$ negative cases with dry eye.

\section{Conflicts of Interest}

The authors declare no conflicts of interest regarding the publication of this paper.

\section{References}

[1] Hessen, M. and Akpek, E.K. (2014) Dry Eye: An Inflammatory Ocular Disease. Journal of Ophthalmic \& Vision Research, 9, 240-250.

[2] Luo, L., Li, D.Q., Doshi, A., Farley, W., Corrales, R.M. and Pflugfelder, S.C. (2004) Experimental Dry Eye Stimulates Production of Inflammatory Cytokines and MMP-9 and Activates MAPK Signalling Pathways on the Ocular Surface. Investigative Ophthalmology \& Visual Science, 45, 4293-4301. https://doi.org/10.1167/iovs.03-1145

[3] Aragona, P., Aguennouz, M., Rania, L., et al. (2015) Matrix Metalloproteinase 9 and Transglutaminase 2 Expression at the Ocular Surface in Patients with Different Forms of Dry Eye Disease. Ophthalmology, 122, 62-71. https://doi.org/10.1016/j.ophtha.2014.07.048

[4] Chotikavanich, S., de Paiva, C.S, de Li, Q., et al. (2009) Production and Activity of Matrix Metalloproteinase-9 on the Ocular Surface Increase in Dysfunctional Tear Syndrome. Investigative Ophthalmology \& Visual Science, 50, 3203-3209. https://doi.org/10.1167/iovs.08-2476

[5] Messmer, E.M., von Lindenfels, V., Garbe, A. and Kampik, A. (2016) Matrix Metalloproteinase 9 Testing in Dry Eye Disease Using a Commercially Available Point-of-Care Immunoassay. Ophthalmology, 123, 2300-2308. https://doi.org/10.1016/j.ophtha.2016.07.028

[6] De Paiva, C.S., Corrales, R.M., Villarreal, A.L., et al. (2006) Corticosteroid and Doxycycline Suppress MMP-9 and Inflammatory Cytokine Expression, MAPK Ac- 
tivation in the Corneal Epithelium in Experimental Dry Eye. Experimental Eye Research, 83, 526-535. https://doi.org/10.1016/j.exer.2006.02.004

[7] Park, J.Y., Kim, B.G., Kim, J.S. and Hwang, J.H. (2018) Matrix Metalloproteinase 9 Point-of-Care Immunoassay Result Predicts Response to Topical Cyclosporine Treatment in Dry Eye Disease. Translational Vision Science \& Technology, 7, 31-34. https://doi.org/10.1167/tvst.7.5.31

[8] Diaz-Llopis, M., Pinazo-Duran, M.D., Diaz-Guiñon, L., Rahhal-Ortuño, M., Perez-Ramos, M., Bosch, R., Gallego-Pinazo, R., Dolz-Marco, R., Diaz-Guiñon, T., Diaz, M., Romero, F.J. and Cisneros, A. (2019) A Randomized Multicenter Study Comparing Seawater Washes and Carmellose Artificial Tears Eyedrops in the Treatment of Dry Eye Syndrome. Clinical Ophthalmology, 13, 483-490. https://doi.org/10.2147/OPTH.S185409

[9] The Definition and Classification of Dry Eye Disease: Report of the Definition and Classification Subcommittee of the International Dry Eye Workshop (2007). The Ocular Surface, 5, 75-92. https://doi.org/10.1016/S1542-0124(12)70081-2

[10] The Epidemiology of Dry Eye Disease: Report of the Epidemiology Subcommittee of the International Dry Eye Workshop. The Ocular Surface, 5, 93-107. https://doi.org/10.1016/S1542-0124(12)70082-4

[11] Sambursky, R., Davitt, W.F., Friedberg, M. and Tauber, S. (2014) Prospective, Multicenter, Clinical Evaluation of Point-of-Care Matrix Metalloproteinase- 9 Test for Confirming Dry Eye Disease. Cornea, 33, 812-818. https://doi.org/10.1097/ICO.0000000000000175

[12] Sambursky, R. (2016) Presence or Absence of Ocular Surface Inflammation Directs Clinical and Therapeutic Management of Dry Eye. Clinical Ophthalmology, 10, 2337-2343. https://doi.org/10.2147/OPTH.S121256

[13] Tong, A.Y., Passi, S.F. and Gupta, P.K. (2020) Clinical Outcomes of Lifitegrast 5\% Ophthalmic Solution in the Treatment of Dry Eye Disease. Eye Contact Lens, 46, S20-S24. https://doi.org/10.1097/ICL.0000000000000601

[14] Bonnomet, A., Luczka, E., Coraux, C. and de Gabory, L. (2016) Non-Diluted Seawater Enhances Nasal Ciliary Beat Frequency and Wound Repair Speed Compared to Diluted Seawater and Normal Saline. International Forum of Allergy \& Rhinology, 6, 1062-1068. https://doi.org/10.1002/alr.21782

[15] Bastier, P.L., Lechot, A., Bordenave, L., Durand, M. and de Gabory, L. (2015) Nasal Irrigation: From Empiricism to Evidence-Based Medicine. A Review. European Annals of Oto-rhino-laryngology, Head and Neck Diseases, 132, 281-285. https://doi.org/10.1016/j.anorl.2015.08.001

[16] Proksch, E., Nissen, H.P., Bremgartner, M. and Urquhart, C. (2005) Bathing in a Magnesium-Rich Dead Sea Salt Solution Improves Skin Barrier Function, Enhances Skin Hydration, and Reduces Inflammation in Atopic Dry Skin. International Journal of Dermatology, 44, 151-157. https://doi.org/10.1111/j.1365-4632.2005.02079.x

[17] Chun, S.Y., Lee, K.S. and Nam, K.S. (2017) Refined Deep-Sea Water Suppresses Inflammatory Responses via the MAPK/AP-1 and NF- $\kappa$ B Signalling Pathway in LPS-Treated RAW 264. International Journal of Molecular Sciences, 18, 11-13. https://doi.org/10.3390/ijms18112282 